\title{
Primer registro de Triatoma nigromaculata (Stål, 1859) (Hemiptera, Reduviidae, Triatominae) para Colombia
}

\author{
Luis Reinel Vásquez ${ }^{1}$, Cleber Galvão ${ }^{2}$, Néstor A. Pinto ${ }^{3}$, Humberto Granados ${ }^{4}$ \\ 1 Centro de Estudios en Microbiología y Parasitología, Departamento de Medicina Interna, Facultad de \\ Ciencias de la Salud, Universidad del Cauca, Colombia. \\ 2 Laboratório Nacional e Internacional de Referência em Taxonomia de Triatomíneos, Departamento de \\ Entomologia, Instituto Oswaldo Cruz, Rio de Janeiro, RJ, Brasil. \\ 3 Centro de Investigaciones en Parasitología y Medicina Tropical, Universidad de los Andes, Bogotá, \\ D. C., Colombia. \\ 4 Facultad Ciencias de la Salud, Universidad del Cauca, Popayán, Colombia.
}

Se registra por primera vez para Colombia la presencia de Triatoma nigromaculata en la vereda La Playa, municipio de El Tambo, departamento del Cauca. Una sola hembra fue capturada en el peridomicilio. No se pudo realizar el examen para determinar la presencia de Trypanosoma debido al mal estado de preservación del ejemplar. Se discute la importancia epidemiológica de esta captura para la transmisión de la enfermedad de Chagas en el departamento del Cauca y en Colombia.

Palabras clave: enfermedad de Chagas, Triatominae, Triatoma, Colombia.

First report of Triatoma nigromaculata (Stål, 1859) (Hemiptera, Reduviidae, Triatominae) for Colombia

The presence of Triatoma nigromaculata was recorded for the first time in Colombia in the rural village La Playa in the province of Cauca. A single female was captured from an outdoor area near a household. The presence of Trypanosoma parasites in the specimen was not established due to the poor condition of the specimen. The epidemiological importance of this finding is discussed with respect to the potential for transmission of Chagas disease in the province of Cauca and, more broadly, in Colombia.

Keywords: Chagas disease, Triatominae, Triatoma, Colombia.

La enfermedad de Chagas es una parasitosis que presenta su distribución en el continente americano y es causada por el hemoparásito Trypanonosoma cruzi. De acuerdo con la Organización Mundial de la Salud (OMS), en este continente se encuentran infectados alrededor de 18 millones de personas. En Colombia se estima que la prevalencia de infección humana por $T$. cruzi es de 1'300.000 individuos $(1,2)$.

Los vectores de la enfermedad son los chinches hematófagos de la subfamilia Triatominae.

Correspondencia:

Luis Reinel Vásquez, Carrera 16 No. 14N-02, Popayán, Colombia.

Teléfono 820 4684, extensión 222

Ireinel@unicauca.edu.co

Recibido: 1712/04; aceptado: 12/05/05
Actualmente son reconocidos 136 nombres válidos agrupados en 18 géneros y 6 tribus (3); se excluyen de la lista de especies un género monotípico Torrealbaia Carcavallo, Jurberg \& Lent, 1998, que según por Forero et al. es sinónimo de Amphibolus Klug, 1839 (Harpactorinae) (4).

En Colombia, Uribe en 1929 (5) informó el hallazgo de $T$. cruzi en ejemplares de Rhodnius prolixus procedentes del departamento del Tolima. Posteriormente, Ucrós en 1960 (6), Marinkelle en 1969 (7), Ucrós et al. en 1971(8) y D'Alesandro et al. en 1971 (9) ampliaron el conocimiento de la distribución de los triatominos en el territorio colombiano. De estas revisiones se destacan la presencia en la región oriental del país de $R$. prolixus, Triatoma dimidiata, T. maculata, $R$. robustus, $R$. brethesi y $T$. venosa distribuidos por 
debajo de los 2.000 msnm. También se destacó la presencia de triatominos silvestres como Panstrongylus geniculatus, $R$. pallescens, Eratyrus cuspidatus, Cavernicola pilosa y Microtriatoma trinidadensis (como M. mansosotoi), distribuidos en la región oriental y occidental.

En 1990, Corredor et al. (10) presentaron un estudio sobre la distribución de triatominos domiciliados en Colombia realizados en las zonas oriental y occidental del país. En esta actualización se observó que en $95,4 \%$ de las viviendas infestadas se encontraba presente $R$. prolixus aunque también se encontró en el intradomicilio $T$. maculata, $T$. dimidiata, $T$. venosa y $P$. geniculatus. De acuerdo con este estudio no se registraron triatominos en las casas en el departamento del Cauca.

Finalmente, se desarrolló de 1998 al 2000 el Programa Nacional de Prevención y Control de la
Enfermedad de Chagas y la Cardiopatia Infantil y se realizó una actualización de los vectores de $T$. cruzi; se estudiaron los departamentos de Antioquia, Córdoba, Bolívar, Cundinamarca, Boyacá, Casanare, Santander, Norte de Santander, Arauca, Meta, Tolima, Magdalena, Sucre, Cesar y La Guajira. En esta encuesta se destacó la mayor distribución de $P$. geniculatus y $R$. prolixus, y se observó la presencia de $E$. mucronatus en el domicilio en Norte de Santander (11-15).

Molina et al. (16) recopiló los hallazgos de otras investigaciones referentes a la situación de triatominos para Colombia. Se estableció para el país la presencia de 23 especies vectoras, 15 de con infecciones naturales con T. cruzi. La publicación resalta las capacidades de transmisión de especies silvestres que son atraídas por la luz de las casas como es el caso

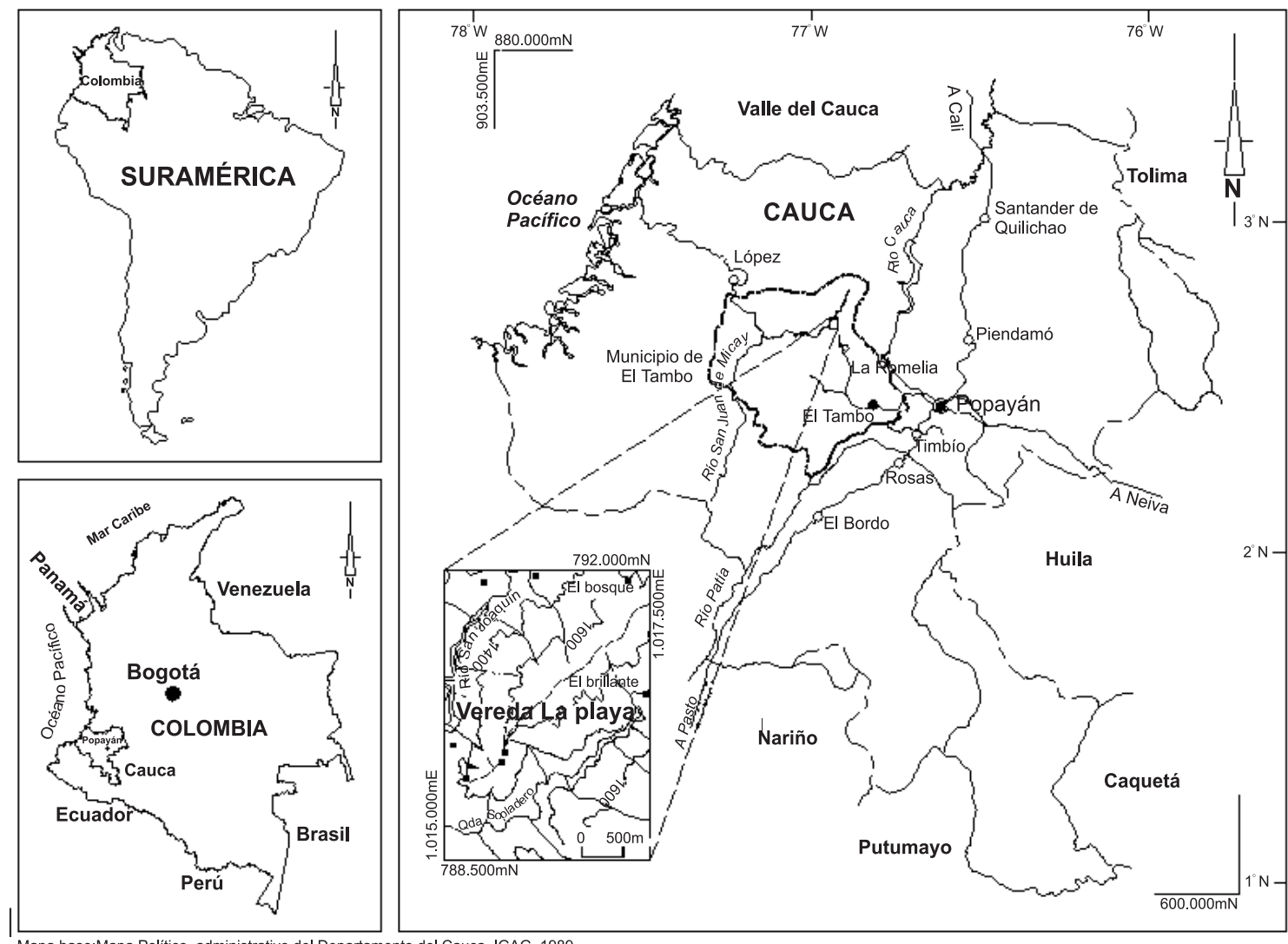

Mapa base:Mapa Político, administrativo del Departamento del Cauca, IGAG, 1989

Figura 1. Localización geográfica de la vereda La Playa, municipio de ElTambo, departamento del Cauca, Colombia 


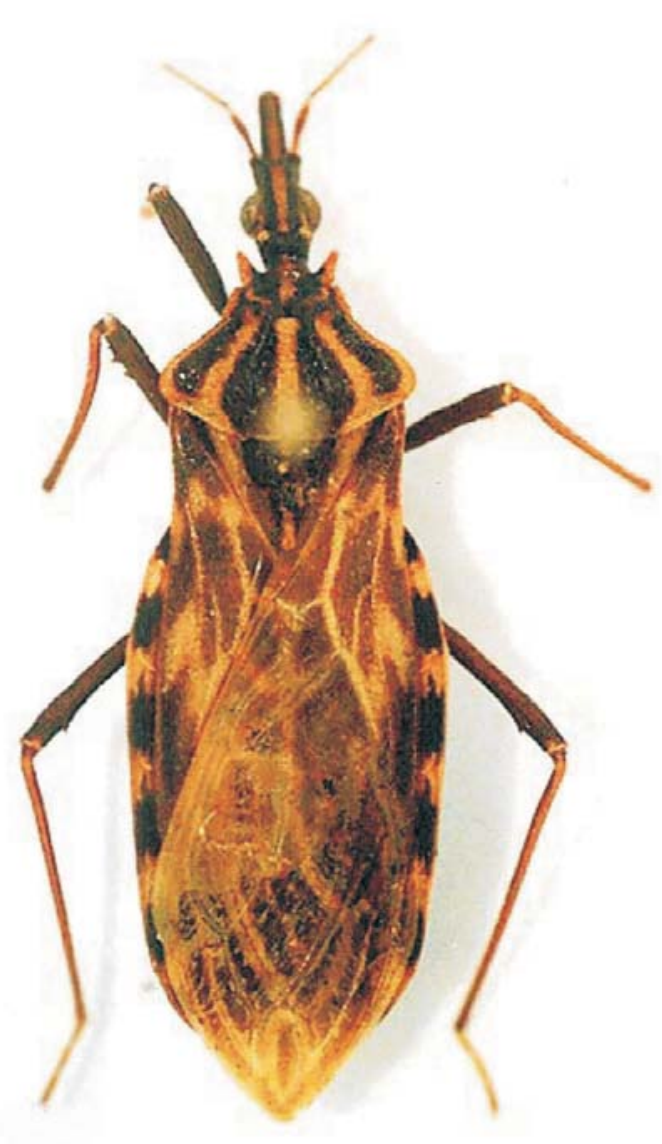

Figura 2. Triatoma nigromaculata (Stål, 1859), ejemplar encontrado en la vereda La Playa del municipio de EI Tambo en Cauca, Colombia.

de $P$. rufotuberculatus, E. cuspidatus, E. mucronatus, $R$. pictipes, $R$. robustus, $R$. dalessandroi, $R$. neivai y $R$. brethesi.

En el departamento del Cauca se ha registrado la presencia de $P$. geniculatus en la isla Gorgona, jurisdicción del municipio de Guapi; esta descripción original fue realizada por Chessman en 1924, según Barreto et al. (17). También se tiene registrado en el Cauca $P$. rufotuberculatus en el municipio de Santander de Quilichao, y se encontró asociado al vampiro Desmodus rotundus e infectado con T. cruzi (18).

El presente trabajo registra por primera vez el hallazgo de T. nigromaculata en la vereda La Playa en el municipio de El Tambo en el departamento del Cauca, Colombia.

\section{Materiales y métodos}

\section{Descripción del área de estudio}

El triatomino se halló en la vereda La Playa, localizada a $2^{\circ} 30^{\prime} \mathrm{N}-76^{\circ} 30^{\prime} \mathrm{W}$; su altura sobre el nivel del mar es de 1600 msnm y está circundada por la quebrada Sopladero y el río San Joaquín. Esta vereda corresponde al caserío de Romelia y esta ubicada al noroeste del municipio de El Tambo distante de Popayán (capital del departamento del Cauca) a $50 \mathrm{~km}$, aproximadamente (figura 1).

El ejemplar fue hallado de forma accidental durante la actividad de captura de ofidios por uno de los autores quien ha realizado salidas de campo en el área desde 1975.

\section{Identificación}

El triatomino fue identificado como T. nigromaculata mediante las claves dicotómicas de Lent \& Wygodzinsky (1979) (19) y por comparación con ejemplares depositados en las colecciones de referencia del Laboratorio Nacional e Internacional de Referencia en Taxonomía de Triatomíneos (LNIRTT), Departamento de Entomología, Instituto Oswaldo Cruz, Río de Janeiro, Brasil.

\section{Resultados}

El ejemplar estudiado es una hembra encontrada en 1999 en la vereda La Playa en el municipio del El Tambo, departamento de Cauca (figura 1), y que debido a que el triatomino se encontraba muy seco no se investigó la presencia de tripanosomatidos. El especimen estaba mal preservado; sin embargo, fue posible identificarlo como $T$. nigromaculata mediante las claves dicotómicas de Lent y Wygodzinsky (1979), y mediante la comparación con especímenes depositados en las colecciones de referencia. La especie es bien característica y las principales estructuras morfológicas diagnósticas y que permiten la diferencia de T. venosa (Stål, 1872), la especie morfológicamente más próxima, son los ángulos anterolaterales del pronoto alargados y las manchas oscuras de los segmentos del conexivo, puntiagudas y similares a forma de bigornia (figura 2).

Esta especie fue capturada entre las hierbas en unos encierros o corrales para animales pequeños de la zona cercana a la vivienda en las horas de 
la mañana con la colaboración de un campesino. Después de esta captura el ejemplar fue entregado en el Centro de Estudios en Microbiología y Parasitología de la Universidad del Cauca e, inicialmente, se pensó que se trataba de $T$. rubida. Sin embargo, para resolver esta discrepancia sobre la ubicación taxonómica del ejemplar se establecieron contactos con investigadores expertos del grupo de Triatominos quienes, finalmente, lo determinaron como T. nigromaculata.

\section{Discusión}

T. nigromaculata es una especie silvestre que se ha encontrado en huecos de árboles $y$, ocasionalmente, en gallineros y domicilios humanos (19). Fue originalmente descrita en Venezuela y, posteriormente, se encontró en una sola ocasión en Perú (20). Scorza et al. encontraron esta especie colonizando domicilios humanos en los Andes venezolanos (21). En la actualización de la distribución geográfica de los triatominos de Galvão et al. se registra en Venezuela y Perú, pero no en Colombia.

Molina et al. en su publicación ya habían nombrado la posibilidad de encontrar a T. nigromaculata en el territorio colombiano pero en el departamento de Norte de Santander (16); los autores registran por primera vez el hallazgo de esta especie en el departamento del Cauca. Esta especie como vector de $T$. cruzi debería ser estudiada epidemiológicamente ya que es posible encontrar ecótopos intervenidos por el hombre y debido a esto el comportamiento del vector (por ejemplo vuelos dispersivos) puede cambiar en la búsqueda de nuevas fuentes de alimento y la adaptación a domicilios humanos pero la situación de orden público y los procesos de reestructuración en secretarías de salud crean un inconveniente en la realización de este tipo de actividades $(2,16,22)$.

Finalmente, el ejemplar examinado está depositado con el número 3042 en la Colección Herman Lent del LNIRTT, perteneciente a la Colección Entomológica del Instituto Oswaldo Cruz, Río de Janeiro, Brasil.

\section{Agradecimientos}

Los autores agradecen a Jorge Enrique García por su colaboración con la serie fotográfica del triatomino. A Felipe Guhl, director del Centro de Investigaciones en Microbiología y Parasitología Tropical-CIMPAT, Universidad de los Andes y a Gustavo Vallejo y Julio C. Carranza del Laboratorio de Investigaciones en Parasitología Tropical de la Universidad del Tolima por su colaboración.

\section{Conflicto de interés}

Los autores del presente escrito declaran que no existe conflicto de intereses.

\section{Financiación}

La financiación fue realizada por el Conselho Nacional de Desenvolvimento Científico e Tecnológico (CNPq), Brasil y del Centro de Estudios de Microbiología y ParasitologíaCEMPA, Facultad Ciencias de la Salud, Universidad del Cauca, Popayán, Colombia.

\section{Referencias}

1. Schmunis GA. Trypanosoma cruzi: etiologic agent of Chagas disease. Status in the blood supply in endemic and non-endemic countries. Transfusion 1991;31:547-57.

2. Guhl F, Angulo V, Restrepo M, Nicholls S, Montoya R. Estado del arte de la enfermedad de Chagas Colombia y estrategias de control. Biomédica 2003;23 (Supl.1):31-7.

3. Galvão C, Carcavallo RU, Rocha DS, Jurberg J. A checklist of the current valid species of the subfamily Triatominae Jeannel, 1919 (Hemiptera, Reduviidae) and their geographical distribution, with nomenclatural and taxonomic notes. Zootaxa 2003;202:1-36.

4. Forero D, Weirauch C, Baena M. Synonymy of the reduviid (Hemiptera: Heteroptera) genus Torrealbaia (Triatominae) with Amphibolus (Harpactorinae), with notes on Amphibolus venator (Klug, 1830). Zootaxa 2004;670:1-12.

5. Uribe C. Infección de Rhodnius prolixus Stahl por Trypanosoma cruzi y T. rangeli. Biomédica 1996;16:8792.

6. Ucrós H. Distribución de los Triatominae en Colombia. Rev Fac Med Bogotá 1960;28:181-9.

7. Marinkelle $\mathbf{C J}$. The distribution of Colombian Triatominae and their infestation with trypanosomatid flagellates. Geneve: World Health Organization; 1969.

8. Ucrós H, Rocha H, Duque M. Distribución de los Triatominae en Colombia. Antioquia Médica 1971; 21:707-17.

9. D'Alessandro A, Barreto P, Duarte CA. Distribution of triatomine-transmitted trypanosomiasis in Colombia and new records of the bugs and infections. J Med Entomol 1971;8:159-72. 
10. Corredor A, Santacruz MM, Páez S, Guatame LA. Distribución de los triatominos domiciliarios en Colombia. Bogotá: Instituto Nacional de Salud; 1990.

11. Guhl F. Estado actual del control de la Enfermedad de Chagas en Colombia. En: Guhl F, Jaramillo CA, editores. Memorias, curso-taller: Control de tripanosomosis americana y leishmaniosis. Aspectos biológicos, genéticos y moleculares. Santafé de Bogotá: Corcas Editores Ltda.: 1998. p.47-81.

12. Restrepo M, Restrepo BN, Salazar CI, Parra GJ. Programa Nacional de Prevención y Control de la Enfermedad de Chagas y la Cardiopatía Infantil. Nodo Occidental: Instituto Colombiano de Medicina Tropical (Antioquia, Cordoba, Bolivar). En: Angulo VM, editor. Memorias, curso-taller internacional. Control y manejo de la tripanosomiasis americana. Bucaramanga: Gráficas Trijaimes; 1999. p.87-93.

13. Guhl F, Barrios D, Cordobés JM, Herrera C, Molina J, Pachón D, Pinto N. Programa Nacional de Prevención y Control de la Enfermedad de Chagas y la Cardiopatía Infantil. Región centro-oriente. Nodo Universidad de los Andes (Cundinamarca, Boyacá y Casanare). En: Angulo VM, editor. Memorias, cursotaller internacional. Control y manejo de la tripanosomiasis americana. Bucaramanga: Gráficas Trijaimes; 1999. p.94-8.

14. Angulo VM, Tarazona Z, Reyes A, Gutiérrez R, Sandoval M. Programa Nacional de Prevención y Control de la Enfermedad de Chagas y la Cardiopatía Infantil. Nodo nororiental, CINTROP-Universidad Industrial de Santander (Arauca, Norte de Santander y Santander). En: Angulo VM, editor. Memorias, cursotaller internacional. Control y manejo de la tripanosomiasis americana. Bucaramanga: Gráficas Trijaimes; 1999. p.99-108.
15. Gutiérrez R, Angulo VM, Aguilar F. Aspectos ecológicos de la enfermedad de Chagas en la región nororiental de Colombia. En: Vallejo GA, Carranza JC, Jaramillo JC, editores. Biología, epidemiología y control de la tripanosomosis americana y leishmaniosis. Ibagué: Lito Ediciones Tolima; 2000. p.33-4.

16. Molina JA, Gualdrón LE, Brochero LH, Olano VA, Barrios D, Guhl F. Distribución actual e importancia epidemiológica de las especies de triatominos (Reduviidae: Triatominae) en Colombia. Biomédica 2000;20:344-60.

17. Barreto $\mathbf{P}$, Barreto $\mathbf{M}$, Hurtado $\mathbf{C}$. Nuevos hallazgos en Colombia de Panstrongylus geniculatus, (Latreille, 1811), y Triatoma dispar Lent 1950 (Hemiptera: Reduviidae). Colombia Médica 1988;19:64-6.

18. D'Alessandro A, Barreto P, Thomas M. Nuevos registros de triatominos domiciliarios y extradomiciliarios en Colombia. Colombia Médica 1981;12:75-85.

19. Lent $\mathbf{H}$, Wygodzinsky. Revision of the Triatominae (Hemiptera, Reduviidae), and their significance as vectors of Chagas disease. Bull Am Mus Nat Hist 1979;163:123-520.

20. Calderón FGE, Monzón L. Nota científica: primer hallazgo de Triatoma nigromaculata (Stål, 1872) en el Perú. Rev Peruana Entomol 1995;37:124.

21. Scorza JV, Solarte Y, Moreno E. The epidemiological importance of Triatoma nigromaculata (Stal, 1859) colonizing human dwellings of the Venezuelan Andes. Mem Inst Oswaldo Cruz 1994;89:299.

22. Castillo D, Wolff M. Aspectos del comportamiento de los triatominos (Hemiptera: Reduviidae), vectores de la enfermedad de Chagas. Biomédica 2000;20:59-64. 